\title{
Occult pulmonary mucosa-associated lymphoid tissue lymphoma presenting as catastrophic antiphospholipid antibody syndrome
}

\author{
HARIHARAN REGUNATH $^{1 *}$, JAMES SHORTRIDGE $^{1 *}$, SHAHZAD RAZA $^{2}$, PUJA NISTALA $^{2}$, \\ BRANDON M. HUFFMAN ${ }^{3}$, MICHAEL X. WANG ${ }^{3}$ and DONG XIANG ${ }^{2}$ \\ ${ }^{1}$ Department of Internal Medicine, University of Missouri; ${ }^{2}$ Division of Hematology and Medical Oncology; \\ ${ }^{3}$ Department of Pathology and Anatomical Sciences, Ellis Fischel Cancer Center, \\ University of Missouri, Columbia, MO 65203, USA
}

Received January 19, 2013; Accepted June 12, 2013

DOI: $10.3892 / 01.2013 .1585$

\begin{abstract}
Catastrophic antiphospholipid antibody syndrome (CAPS) is characterized by fulminant thrombosis of the arterial and venous beds of multiple organ systems over a relatively short period of time and with a high mortality rate. Mucosa-associated lymphoid tissue (MALT) lymphoma of the lung has never been reported as a causative or precipitating factor for CAPS in the CAPS registry database. The present study describes a rare case of pulmonary MALT lymphoma of the lung that presented as CAPS. A 19-year-old Hispanic female presented with shortness of breath and abdominal pain. Computed tomography (CT) scans of the chest and abdomen revealed multiple portal vein thromboses and bilateral pulmonary nodules. Within one week of presentation, the patient developed a straight sinus thrombosis and upper extremity deep vein thrombosis, which led to shortness of breath. A biopsy of the lung nodule revealed MALT lymphoma. The present case illustrates a rarely reported pulmonary MALT lymphoma presenting as CAPS in a young female. The patient was successfully treated with $90 \mathrm{mg} / \mathrm{m}^{2}$ bendamustine on days one and two and rituximab $375 \mathrm{mg} / \mathrm{m}^{2}$ on day one of each 28-day cycle. Complete remission of the lung nodules was observed following three cycles of treatment, as visualized by positron emission tomography (PET)/CT scan. Fondaparinux was identified as a feasible anticoagulation drug of choice for this case. At seven months post-treatment, the patient continues to be stable with no further evidence of thrombosis and is currently undergoing rituximab maintenance therapy every six months for two years. A repeat lupus anticoagulant
\end{abstract}

Correspondence to: Dr Dong Xiang, Ellis Fischel Cancer Center, University of Missouri, 115 Business Loop 70 E, Columbia, MO 65203, USA

E-mail: xiangdo@health.missouri.edu

*Contributed equally

Key words: lymphoma, antiphospholipid antibody, rituximab, plasmapheresis antibody assay turned and remained negative during the clinical follow-up period. A prompt diagnosis and early aggressive treatment is potentially curative and may dramatically decrease the mortality risk. Future studies should explore the role of rituximab in the management of CAPS-associated B-cell lymphoid malignancies.

\section{Introduction}

Catastrophic antiphospholipid antibody syndrome (CAPS) is a life-threatening variant of antiphospholipid antibody syndrome (APLA) (1). The condition is typically characterized by fulminant thrombosis of the arterial and venous beds of multiple organ systems over a relatively short period of time. Although it has been reported to occur in a small percentage of patients with APLA syndrome, the cognizance of this condition is vital, as early treatment with anticoagulation therapies, aggressive immunosuppression or plasmapheresis may decrease morbidity and mortality rates $(1,2)$. The present study describes a case of mucosa-associated lymphoid tissue (MALT) lymphoma of the lung that presented as CAPS and was successfully treated using a combination of plasmapheresis, rituximab and fondaparinux anticoagulation, leading to a resolution of a life-threatening event. Written informed consent was obtained from the patient.

\section{Case report}

A 19-year-old Hispanic female with a past history of Evan's syndrome was referred to the University of Missouri Hospital (Columbia, MO, USA) with abdominal pain associated with fever, nausea, vomiting, coughing and hematochezia. A diagnosis of a portal vein thrombosis was confirmed using an abdominal computed tomography (CT) scan (Fig. 1A) and duplex ultrasonogram one day prior to the presentation to the hospital. On admission, the patient was dyspneic with $96 \% \mathrm{SPO}_{2}$ in 21 of oxygen. A full dose of enoxaparin was administered. A contrast chest CT was negative for pulmonary emboli, but disclosed multiple bilateral lung nodules that were distributed peripherally. The nodules measured $1.9 \times 1.8 \mathrm{~cm}$ in the right upper lobe, $2.1 \times 0.9 \mathrm{~cm}$ in the left upper lobe and $1.2 \times 1.1 \mathrm{~cm}$ in the right lower lobe. A small amount of bowel 

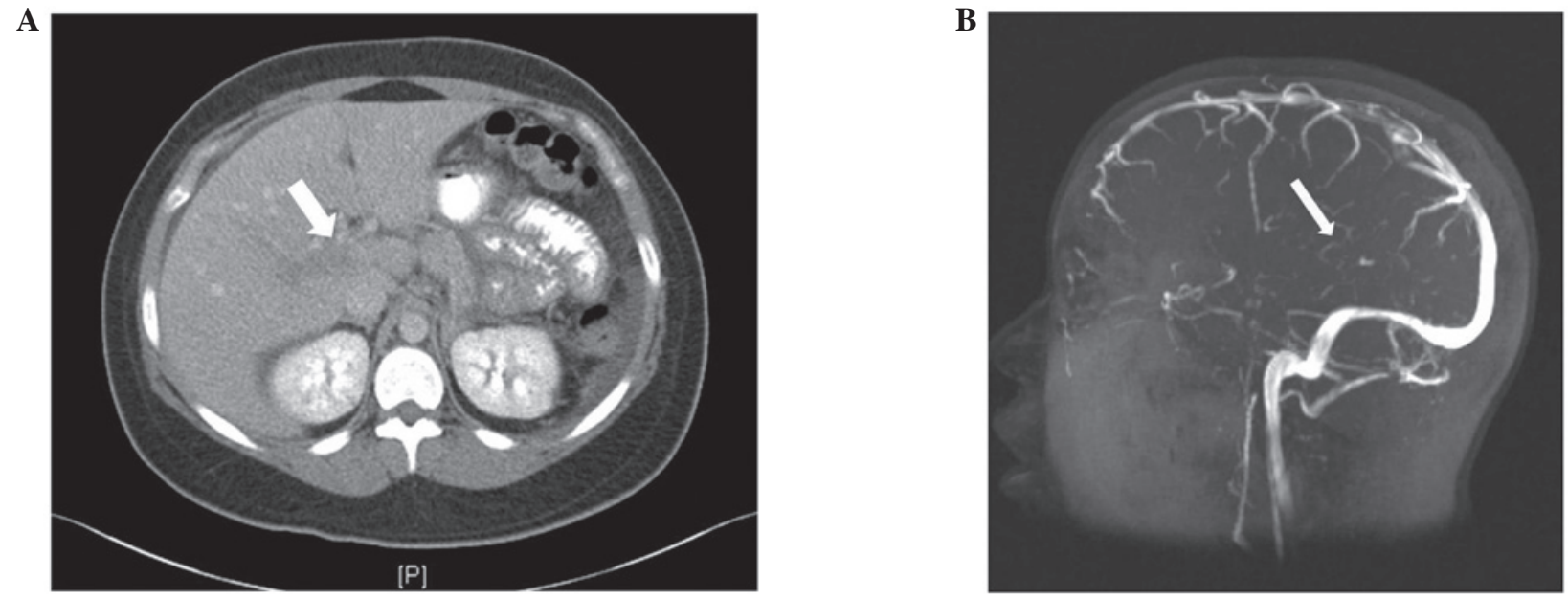

Figure 1. (A) Contrast abdominal CT scan showing a hypodensity and the absence of intravenous contrast noted in the area of the portal vein (white arrow). (B) MRV showing the absence of significant flow in the straight sinus, indicating a thrombosis (white arrow). CT, computed tomography; MRV, magnetic resonance venography.

thickening was noted on the external CT scan. The upper and lower endoscopies were unremarkable, with the exception of the presence of gastropathy. The immunological work-up was positive for the lupus anticoagulant.

On day four, the patient experienced blurred vision in the left eye due to bilateral papilledema, as revealed by an ophthalmoscopic exam. Brain magnetic resonance imaging (MRI) showed a T2/fluid-attenuated inversion recovery (FLAIR) high-signal abnormality involving the left temporal lobe. Within the next $24 \mathrm{~h}$, the symptoms of abdominal pain, hematochezia and headache worsened. The brain MRI was repeated using magnetic resonance venography (MRV), which revealed a thrombosis of the straight sinus (Fig. 1B) with a probable involvement of the veins of Rosenthal, which drain the temporal lobes. A sub-acute venous infarct in the left temporal lobe was also observed. A contrast CT scan of the abdomen revealed a new colonic wall thickening with a notable extension of the portal vein thrombus into the superior mesenteric vein and further caudally, placing the patient at a high risk for ischemic bowel necrosis. Due to the risk of bowel gangrene, following the correction of the international normalized ratio (INR), an exploratory laparotomy was attempted, revealing $40 \mathrm{~cm}$ of necrotic ischemic bowel with no evidence of vasculitis. CAPS was suspected based on the development of venous thromboses in the brain, portal vein and intestine, with a positive lupus anticoagulant assay in a short time-span. The patient was initially administered i.v. heparin and plasmapheresis without steroids due to the concern over wound healing issues.

On the sixth post-operative day, the patient experienced severe hematemesis and hematochezia, therefore, i.v. heparin was stopped. Emergent endoscopies did not reveal a definite source of the bleeding. A repeat abdominal CT scan showed the presence of an ileus and a small bowel lesion, suggesting a developing hematoma. However, the patient was managed conservatively and the condition improved. Four days later, deep vein thromboses developed in the left axillary, brachial and basilic veins. Heparin was restarted using a modified heparin procedure with a lower target partial thromboplastin time (PTT; 40-60 sec) compared with the usual institutional
PTT range $(60-85 \mathrm{sec})$. The range was tolerated without further bleeding or thrombosis. The PTT goal was then gradually increased to a range of $60-85 \mathrm{sec}$ when the patient had been clinically stable for $48 \mathrm{~h}$ on the lower target range.

To treat the suspected CAPS, the patient was administered a non-steroid immunosuppressant monoclonal antibody, rituximab, to avoid poor post-operative wound healing issues from steroid use. The patient continued to improve and was therefore discharged following three weeks of treatment. The patient underwent a percutaneous biopsy of a persistent right lower lobe lung nodule one month after being discharged. Histopathologically, the sections revealed lung parenchyma with bronchial epithelium and vessels that were heavily infiltrated by monocytoid small lymphocytes (Fig 2A). The lymphoid cells were positive for PAX5 (Fig. 2B), BCL2 (Fig. 2C), CD43 (Fig. 2D) and $\kappa$-light chain (Fig. 2E) immunostaining, and were negative for $\lambda$-light chain (Fig. $2 F$ ) immunostaining. The findings were consistent with a diagnosis of extranodal marginal zone lymphoma of respiratory MALT lymphoma.

The patient was treated with bendamustine at $90 \mathrm{mg} / \mathrm{m}^{2}$ on days one and two and $375 \mathrm{mg} / \mathrm{m}^{2}$ rituximab on day one of every 28-day cycle. Complete remission of the lung nodules was observed following three cycles of treatment, as visualized by positron emission tomography (PET)/CT scans. The anticoagulation regime was switched from enoxaparin to fondaparinux. The patient continues to be stable with no further evidence of thrombosis following seven months of treatment and is currently on rituximab maintenance therapy every six months for two years. A repeat lupus anticoagulant antibody assay turned and remained negative in the clinical follow-up appointments.

\section{Discussion}

CAPS is a rare but serious complication of APLA that carries a significantly high mortality rate $(50 \%)(2,4)$. According to the CAPS registry database, the disease is three times more common in females and is usually seen in patients aged $\sim 40$ years old (2). In cases from the CAPS registry, a precipi- 
A

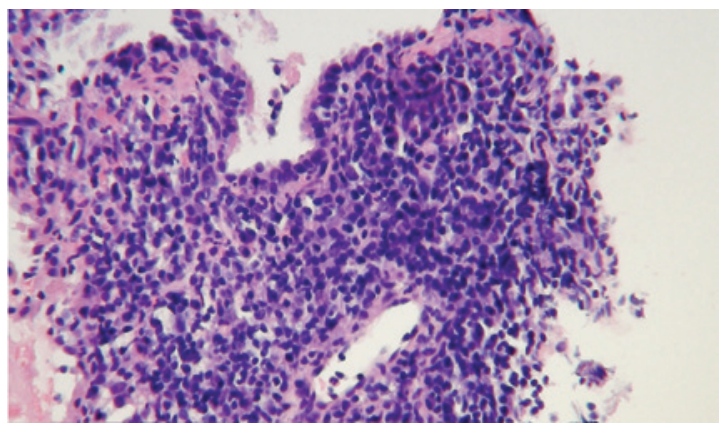

C

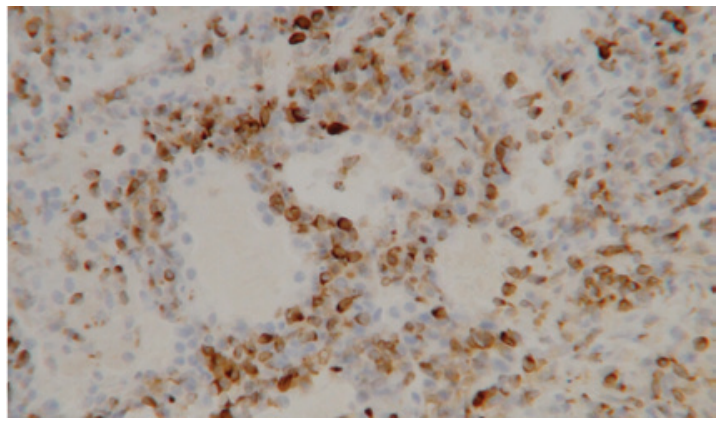

E

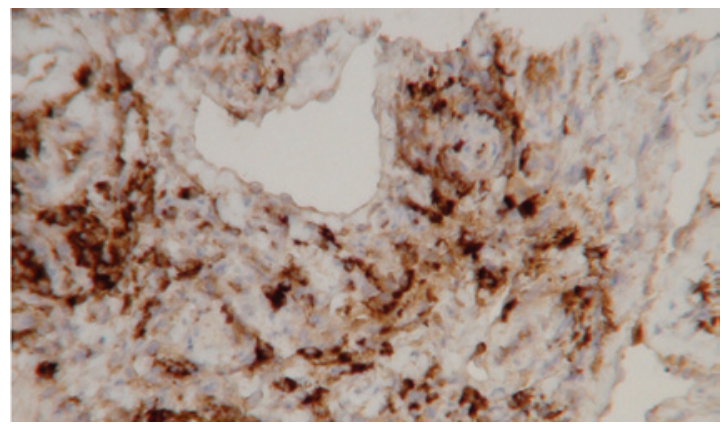

B

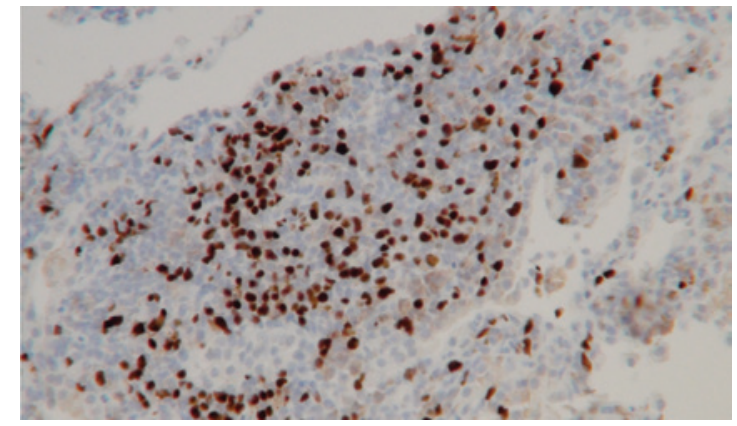

D

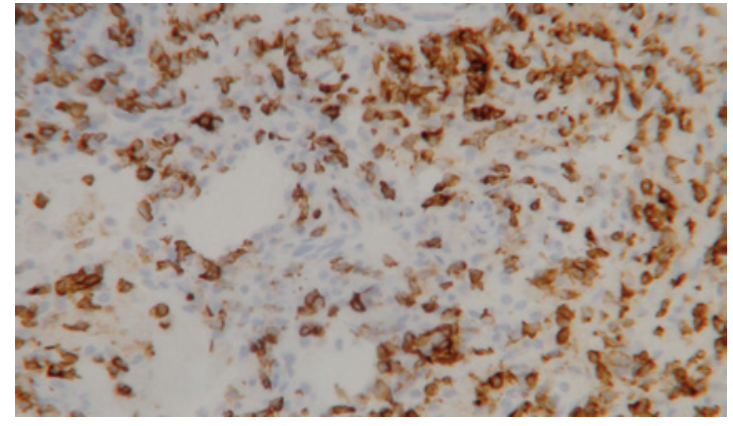

F

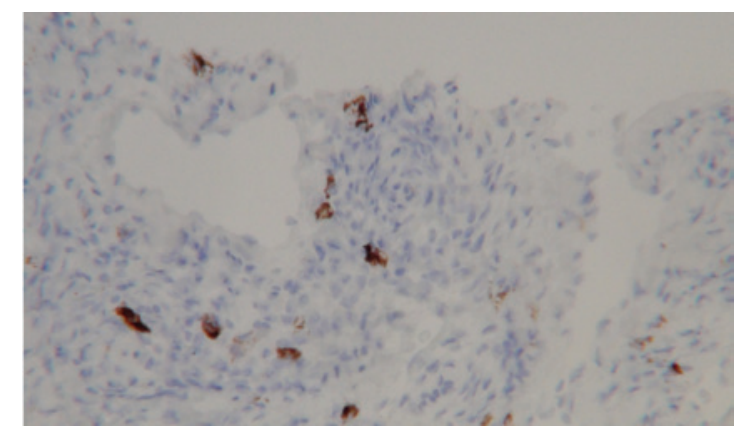

Figure 2. (A) Lymphoepithelial lesion with disruption of the bronchial epithelium and vessel by monocytoid lymphoma cells (H\&E staining; original magnification, x50). (B) PAX5 immunostain highlights small B-cell lymphocytes infiltrating the epithelium (original magnification, x50). (C) Lymphoma cells were BCL2-positive and infiltrated the bronchial epithelium (IHC; original magnification, x50). (D) Lymphoma cells with aberrant expression of CD43 in

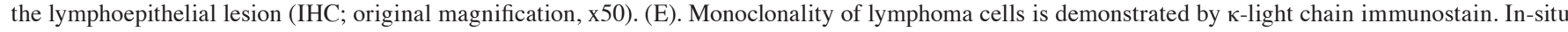
hybridization study confirmed monotypic expression of $\kappa$ immunoglobulin light chains. (IHC; original magnification, $x 50$ ). (F) Sparse immunostain of $\lambda$ immunoglobulin light chain (IHC; original magnification, x50). IHC, immunohistochemistry; H\&E, hematoxylin and eosin.

tating factor was noted in $53 \%$ of cases with infections $(22 \%)$, with recent surgery (10\%) being the most common. Malignancy accounted for $9 \%$ of cases. Hematological malignancies were observed to be the most common (26\%), followed by lung (17\%) and colon (9\%) cancer (2). A pulmonary MALT lymphoma has never been reported as a causative or precipitating factor for CAPS from this registry.

Although a clearly proposed criteria for the diagnosis of non-catastrophic APLA and CAPS exists, the two conditions represent opposite ends of the disease spectrum $(5,6)$. The majority of cases in the CAPS registry were considered 'probable CAPS' primarily due to the lack of a tissue biopsy, which was avoided due to the requirement to stop anticoagulation treatment. The patient in the present study developed a thrombosis of the portal vein, dural venous sinuses and upper extremity deep veins. For the same reason as previously mentioned, a tissue biopsy was not obtained from the liver or lung to confirm the small vessel thrombosis that were shown with clear evidence on imaging studies.

Traditionally, plasmapheresis has been used for CAPS therapy $(7,8)$. In recent years, the addition of rituximab to anticoagulants, steroids and plasmapheresis, has resulted in a reduction of the lupus anticoagulant levels back to normal. In non-catastrophic primary and secondary APS, rituximab therapy has improved the outcome. Four published cases have had a history of B-cell non-Hodgkin lymphoma (NHL), with two being large B-cell lymphomas and two being splenic marginal zone lymphomas $(9,10)$.

Managing anticoagulation itself is very challenging in the event of CAPS. In the situation of an acute bleed that requires a transfusion, it is difficult to continue anticoagulation treatment and therefore, the patient faces the risk of thrombosis. In the present study, when anticoagulation treatment was stopped following the episode of hematemesis, the patient developed an axillary vein thrombosis within 24 hours. Compared with the institutional range of 60-85 sec, the lower target PTT (40-60 sec) strategy was well-tolerated without further bleeding or thrombosis. Such individualization of anticoagulation strategies may be required for managing CAPS where the coagulation system is in labile balance.

The present case is unique in that the age of presentation was younger than usual for malignancy-associated CAPS and 
also that CAPS was the presenting symptom, unlike those cases from the registry and the available studies where all cases had a proven prior history of cancer. CAPS should be suspected in any patient with clinical or radiological evidence of rapidly developing thromboses in multiple organs. The decision to treat the condition using aggressive measures should not be delayed until all criteria are met.

A thrombotic event that is associated with non-gastric MALT lymphoma may be the first manifestation of CAPS. A prompt diagnosis and early aggressive treatment is potentially curative and may dramatically decrease the mortality risk. Rituximab may be an effective adjuvant treatment for managing primary pulmonary MALT lymphoma when combined with bendamustine and fondaparinux. Future studies should explore the role of rituximab in the management of CAPS-associated B-cell lymphoid malignancies.

\section{References}

1. Cervera R, Bucciarelli S, Plasín MA, et al; Catastrophic Antiphospholipid Syndrome (CAPS) Registry Project Group (European Forum On Antiphospholipid Antibodies) Catastrophic antiphospholipid syndrome (CAPS): descriptive analysis of a series of 280 patients from the 'CAPS Registry'. J Autoimmun 32: 240-245, 2009.
2. Miesbach W: Malignancies and catastrophic anti-phospholipid syndrome. Clin Rev Allergy Immunol 36: 91-97, 2009.

3. Rawal A, Finn WG, Schnitzer B and Valdez R: Site-specific morphologic differences in extranodal marginal zone B-cell lymphomas. Arch Pathol Lab Med 131: 1673-1678, 2007.

4. Bucciarelli S, Espinosa G and Cervera R: The CAPS Registry: morbidity and mortality of the catastrophic antiphospholipid syndrome. Lupus 18: 905-912, 2009.

5. Miyakis S, Lockshin MD, Atsumi T, et al: International consensus statement on an update of the classification criteria for definite antiphospholipid syndrome (APS). J Throm Haemost 4: 295-306, 2006.

6. Asherson RA, Cervera R, de Groot PG, et al; Catastrophic Antiphospholipid Syndrome Registry Project Group: Catastrophic antiphospholipid syndrome: international consensus statement on classification criteria and treatment guidelines. Lupus 12: 530-534, 2003.

7. Asherson RA: The catastrophic antiphospholipid (Asherson's) syndrome. Autoimmun Rev 6: 64-67, 2006.

8. Rubenstein E, Arkfeld DG, Metyas S, et al: Rituximab treatment for resistant antiphospholipid syndrome. J Rheumatol 33: 355-357, 2006.

9. Khattri S, Zandman-Goddard G and Peeva E: B-cell directed therapies in antiphospholipid antibody syndrome - new directions based on murine and human data. Autoimmun Rev 11: 717-722, 2012.

10. Ramos-Casals M, Brito-Zerón P, Muñoz S and Soto MJ; BIOGEAS STUDY Group: A systematic review of the off-label use of biological therapies in systemic autoimmune diseases. Medicine (Baltimore) 87: 345-364, 2008. 